\title{
Solar Flares and Related Coronal Magnetic Field Structures in NOAA 8100
}

\author{
Y. Yan \\ Beijing Astronomical Observatory/National Astronomical Observatories, \\ Chinese Academy of Sciences, Beijing 100012, China
}

\begin{abstract}
In this paper we describe the available methods for the nonconstant- $\alpha$ force-free field reconstruction and study the associations between reconstructed $3-\mathrm{d}$ coronal fields by a new technique and the $2 \mathrm{~B} / \mathrm{X} 2$ flare process from observations in AR 8100 on 4 November 1997.
\end{abstract}

\section{Introduction}

Coronal magnetic fields plays a central role in the solar flare process. It is believed that the flare is caused by fast releasing of pre-stored magnetic energy. The rapid conversion of this energy into the kinetic energy of hot plasma and accelerated particles may in turn cause hazardous effects in interplanetary space. Extrapolation is presently still the primary way to reconstruct 3-d coronal field (McClymont et al. 1997, Wang 1999). In this paper we introduce the available methods for non-constant- $\alpha$ force-free field (NLFFF) reconstructions and study the associations between extrapolated 3-d coronal fields by a new boundary integral equation technique (Yan \& Sakurai 1997, 2000) and the flare process from ground and space observations.

\section{On Coronal Magnetic Field Modeling}

There are in general three steps in dealing with any physical problem by mathematical modeling: (1) establishing the mathematical model of the physical problem; (2) solving the mathematical model; (3) explaining the physical meaning of the solution.

Three issues is involved in dealing with a mathematical model, or the corresponding boundary value problem (BVP): (a) the existence of the solution; (b) the uniqueness of the solution; (c) the stability of the solution with respect to the boundary conditions. If we get positive answers to all these questions, the BVP is well-posed. Otherwise, it is ill-posed.

For the NLFFF problem in open space above the Sun, it is, unfortunately, an ill-posed problem up to now. In the literature, however, a NLFFF model is often said well-posed, or not, only with respect to the third item, without knowing anything about the first two items.

The first practical method employing observed boundary data was a finite element method based on the variational principle of NLFFF (Sakurai 1981). The method has the limitation of slow convergence in highly non-potential situ- 


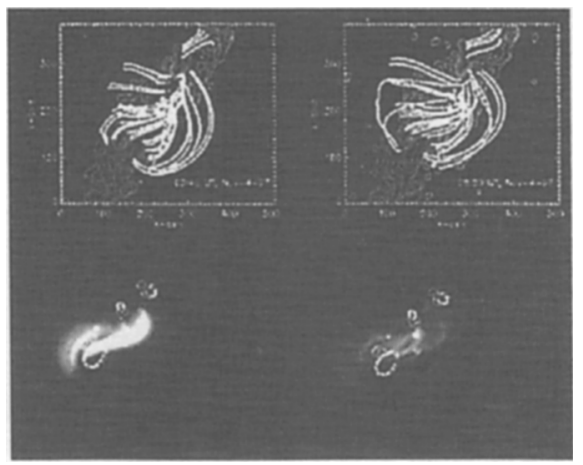

Figure 1. Magnetic field lines (at 03:46 UT) before (a:upper-left) and (at 08:00 UT) after (b:upper-right) the 2B/X2 flare at 05:59 UT. The Yohkoh SXT images at 03:23:26UT (c:lower-left) and at 08:03:44UT (d:lower-right) are shown in the lower panels.

ations (McClymont et al. 1997). A direct discretization of the NLFFF equations by the finite difference techniques (Wu et al. 1990) yields a well-known ill-posed feature for exponentially divergent component cannot be eliminated (Sakurai 1989, McClymont et al. 1997). Amari et al. (1999) improved this ill-poseness using vector potential. Others choose to quasi-physically evolve the MHD equations to a force-free state approximately by temporal evolution instead of a non-linear iteration procedure (Mikić \& McClymont 1994, Roumeliotis 1996). In this case, the huge code and supercomputer resources are in general involved.

All the above methods are based on the differential techniques. On the other hand, a boundary integral equation representation has been recently found, for the first time, for NLFFF in the exterior of the Sun (McClymont et al. 1997, Yan \& Sakurai 1997, 2000). This method has advantages that the solution has a finite energy content in open space above the Sun, or B $\sim o\left(1 / r^{2}\right)$ (Yan \& Sakurai 1997,2000$)$. It follows that if $\alpha$ is consistently determined from the boundary condition and all field lines are rooted there, the NLFFF solution is unique if it exists (Yan 1998, Wang 1999). Under these situations the numerical errors in space are confined by that on the boundary (Yan 1998).

\section{On NOAA 8100 Flare event on 4 November 1997}

Applications have proved that Yan \& Sakurai's NLFFF method is practical and effective. Here we analyze the 2B/X2 flare on 4 November 1997 at 05:58 UT in NOAA 8100.

The 3-d magnetic field structures about 2 hours before (after) the energetic 2B/X2 flare at 05:58 UT are shown in Figure 1. From calculated field lines, that align with the coronal SXR loops properly, apparent twist changes can be observed. However, similar magnetic connectivity has been obtained for both instants, indicating that the magnetic configuration was not significantly reconstructed after the energetic flare. The onset of the flare was at the common footpoints of several interacting magnetic loops and confined near the footpoints 


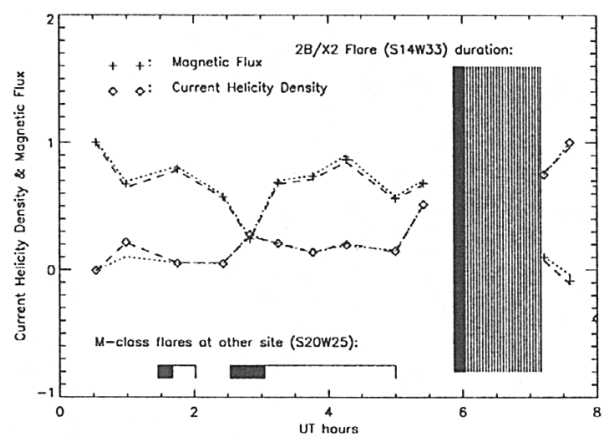

Figure 2. The evolution of the flux $\psi$ ("+") and the averaged current helicity density $\bar{h}_{c}$ (" $\diamond$ ") over the positive polarity patch on 4 November 1997 (The $-1-\sigma$ curves are also plotted in dotted lines). The duration of the energetic flares is marked as dark patches.

of a positive emerging flux region (EFR) in the central upper area of Figure $1(\mathrm{a}, \mathrm{b})$ (Yan et al. 2000).

Figure 2 shows the evolution of the current helicity density and flux at the EFR area (where $\psi=\int_{S} \mathbf{B} \cdot d \mathbf{S}$ and $\bar{h}_{c}=\int_{S} \mathbf{B} \cdot(\alpha \mathbf{B}) d S / \int_{S} d S$ under NLFFF condition, $S$ is the EFR area with its normal upward). They are normalized with respect to their maximum values, $\psi_{\max }=3 \times 10^{21} \mathrm{Mx}$ and $\bar{h}_{\text {cmax }}=0.23 \mathrm{G}^{2} / \mathrm{m}$, respectively. The patch area is $3.5 \times 10^{14} \mathrm{~m}^{2}$. The duration of the $2 \mathrm{~B} / \mathrm{X} 2$ flare is marked in shadow, with dark patches indicating the GOES X-ray eruption duration. It can be seen that during the $2 \mathrm{~B} / \mathrm{X} 2$ flare there is a decrease of the flux across the patch area with positive polarity and there is an increase in the averaged current helicity density over this patch before the onset of the flare.

Acknowledgments The work was supported by NNSF of China (Nos. 49990452, 19973008, 19973016) and Chinese Academy of Sciences (STORM23). Huairou members and Yohkoh team are acknowledged for the data used.

\section{References}

Amari, T., Boulmezaoud, T. Z., and Mikić, Z. 1999, A\&A, 350, 1051

McClymont, A. N., Jiao, L., and Mikić, Z. 1997, Solar Phys., 174, 191

Mikić, Z. and McClymont, A. N. 1994, in A.S.P. Conf. Ser., Vol.68, p. 225

Roumeliotis, G. 1996, ApJ, 473, 1095

Sakurai, T. 1981, Solar Phys., 69, 343

Wang, J. X. 1999, Fundamentals of Cosmic Physics, 20, 251

Wu, S. T., et al. 1990, ApJ, 362, 698

Yan, Y. 1998, Publ. Beijing Astron. Obs., No.4, 66

Yan, Y., Liu, Y., Akioka, M., and Wei, F. 2000, Solar Phys., in press

Yan, Y., and Sakurai, T. 1997, Solar Phys., 174, 65

Yan, Y., and Sakurai, T. 2000, Solar Phys., 195, 89 